\title{
Low-cost $\alpha+\beta$ PM Ti Alloys by Fe/Ni Addition to pure Ti
}

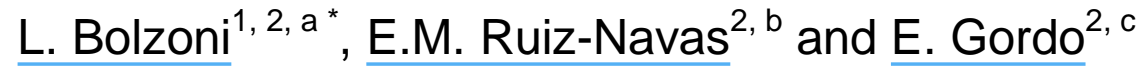 \\ ${ }^{1}$ WaiCAM (Waikato Centre for Advanced Materials), The University of Waikato, Private bag 3105, \\ 3240 Hamilton - New Zealand \\ ${ }^{2}$ Department of Materials Science and Engineering, University Carlos III of Madrid, Avda. de la \\ Universidad, 30, 28911 Leganes, Madrid - Spain \\ a,"leandro@waikato.ac.nz, bemruiz@ing.uc3m.es, elena.gordo@uc3m.es
}

Keywords: Elemental titanium, Powder metallurgy, Pressing and sintering, Low-cost.

\begin{abstract}
Ti and its alloys can deliver a very interesting combination of properties such as low density, high strength, corrosion resistance and biocompatibility and, therefore, are very flexible materials which can be adapted to various applications. Nonetheless, $\mathrm{Ti}$ and $\mathrm{Ti}$ alloys are only employed in critical applications (i.e. aeronautical and aerospace, nautical, medical, etc.) or in products for leisure. In both of these cases the higher fabrication costs of $\mathrm{Ti}$ in comparison to its competitors (i.e. steel and aluminium) is not the limiting factor as it is for many structural applications, especially for mass production (i.e. automotive sector). The use of creative techniques and the decrement of the starting price of Ti have been identified as the two main routes to follow to decrease the fabrication costs. In this study, the production of low-cost $\alpha+\beta \mathrm{Ti}$ alloys has been assessed by combining the addition of cheap alloying elements (in particular a $\mathrm{Fe} / \mathrm{Ni}$ powder) with the classical powder metallurgy route (pressing and sintering). Physical and mechanical properties as well as microstructural analysis of these low-cost alloys were measured and correlated to the processing parameters used to sinter them. It is found that the low-cost $\mathrm{Ti}$ alloys show similar behaviour to conventional $\alpha+\beta$ Ti alloys and, thus, have the potential to be used for non-critical applications.
\end{abstract}

\section{Introduction}

Reduction of the greenhouse pollution, lowering the amount of scrap produced during the fabrication of components and diminishing the number of processing steps are important aspects currently taken into account by the manufacturing sector [1]. Another key feature generally considered during the development and/or improvement of engineered products is the reduction of weight. Consequently, in the last decades, much attention was focused on the employment and optimisation of light metals. Among them, Ti presents a very interesting combination of physical and mechanical properties [2]. In particular, Ti has low density and high strength, and thus noteworthy specific mechanical properties, excellent corrosion resistance and good biocompatibility, because it is a bio-inert material when in contact with the fluids of the human body. The physical metallurgy of $\mathrm{Ti}$ is rather challenging [3] because this metal has a hexagonal crystal structure at room temperature, which results is low deformability, it has high affinity for interstitials (which complicates both its extraction and its processing) and it has low thermal and electrical conductivity which affect its processability. Due to these issues, the application of Ti has always been confined to markets where high production costs are justified by other factors, such as safety in the aerospace sector or reliability in biomedical implants and prostheses.

In recent years, $\mathrm{Ti}$ and its alloys have also been used due to their appealing appearance for architectural components, such as for the façade of the Guggenheim Museum in Bilbao, and leisure goods like watches where mechanical properties are not the most important aspect [2].

In the case of the Ti industry, the achievement of the goals of the manufacturing sector (i.e. low emissions, scraps, etc.) can be pursued by implementing and optimising alternative production routes, by the development of a new generation of (cheaper) alloys with adequate performances or by the combination of these two aspects [4]. Powder metallurgy (PM) techniques have a great 
potential because they are, primarily, solid-state near net shape methods [5]. The fact that the metal does not reach the liquid state is very attractive for $\mathrm{Ti}$ because its reactivity significantly increases with the temperature and, therefore, the interaction with fabrication tools is limited. Furthermore, sedimentation phenomenon due to density difference and solubility limits are not the main restricting factors in the design of novel compositions. Being near net shape methods, PM techniques are characterized by a very high yield of material and do not need as much machining as wrought products, if any is required. Both aspects are very important when speaking of expensive raw materials.

In this work, the cheapest PM process known as cold uniaxial pressing and (vacuum) sintering was applied to assess the viability of producing low-cost $\alpha+\beta$ PM Ti alloys by using cheaper elements with respect the conventional $\beta$ stabilisers (i.e. vanadium, niobium, etc.), specifically using a Fe/Ni (85-15 wt.\%) powder. Iron is not a completely new alloying element to Ti [6-8], but there is still much potential and understanding to gain, especially in PM processing.

\section{Experimental Procedure}

The raw materials for the study were: hydride-dehydride (HDH) elemental Ti powder (purity > $99.8 \%$ and particle size $<90 \mu \mathrm{m})$ and an electrolysed $\mathrm{Fe} / \mathrm{Ni}(85-15)$ powder (particle size $<10 \mu \mathrm{m}$ ). Raw materials were used to fabricate two compositions: Ti-5.41Fe/Ni and Ti-7.57Fe/Ni (whose $\mathrm{Fe}$ content is $5 \mathrm{wt} . \%$ and $7 \mathrm{wt} . \%$, respectively, from the Molchanova equation [9]) by mixing by means of a Turbula mixer. The morphology and particle size distribution of the starting powders were measured prior compaction of green samples. Compacts were shaped by means of a floating die (lubricated with zinc stearate) applying $700 \mathrm{MPa}$ through a uniaxial press. The sintering temperature used ranged between $900^{\circ} \mathrm{C}$ and $1300^{\circ} \mathrm{C}$, dwell time was $1 \mathrm{~h}$, heating and cooling were done at $5^{\circ} \mathrm{C} / \mathrm{s}$ and sintering was carried out under high vacuum $\left(10^{-5} \mathrm{mbar}\right)$. The effects of the sintering step were quantified by measuring the variation of the density (assessed by Archimedes method) and the level of porosity. Samples were prepared for metallographic analysis using the classical route and were etched with Kroll reactant. Vickers hardness was measured on the crosssection of polished samples.

\section{Results and Discussion}

Fig. 1 shows the SEM micrographs of the starting materials where it can be seen that the morphology of the HDH Ti powder is irregular or angular, because the powder was obtained via a comminution process. The $\mathrm{Fe} / \mathrm{Ni}(85-15)$ powder is characterised by a spherical morphology produced by means of the electrolytic route although it can be seen that there are many particles with satellites small particles as well as some agglomeration of irregular particles. The irregular morphology of the HDH Ti powder, which constitutes the great majority of the Ti-5.41Fe/Ni and Ti7.57Fe/Ni alloys, provides the skeleton and the strength to the green samples for handling.
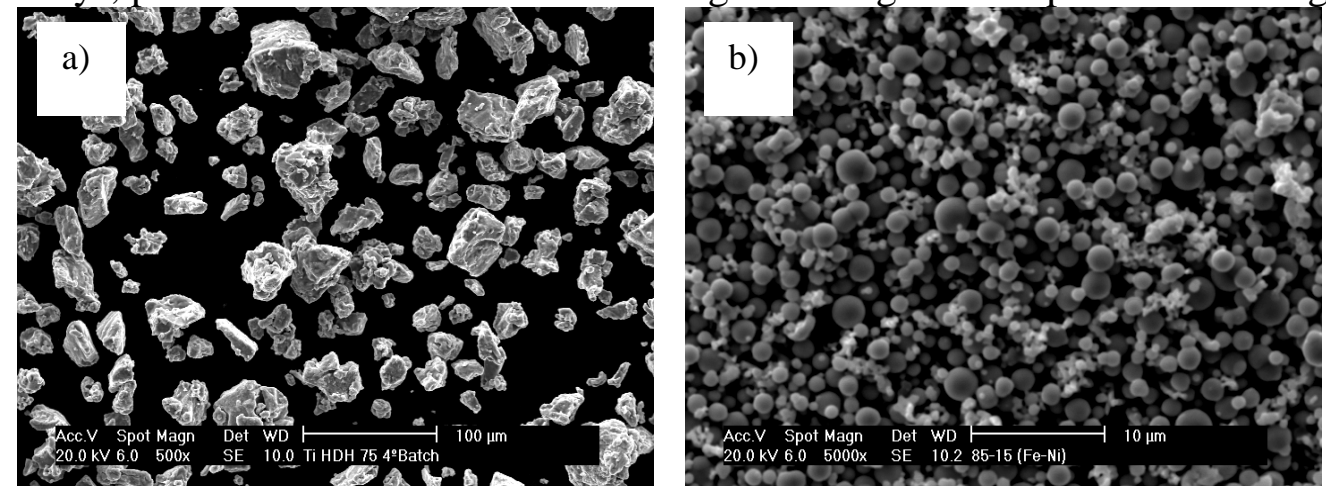

Figure 1 SEM images of the morphology of the raw materials used for the study: a) irregular HDH $\mathrm{Ti}$ and b) spherical Fe/Ni (85-15) powder

The Fe/Ni (85-15) powder particles should fit in between the Ti particles achieving high green 
density values. The very fine particle size of the $\mathrm{Fe} / \mathrm{Ni}$ (85-15) powder was chosen in order to favour diffusion of the alloying elements into the Ti matrix as well as the densification of the materials, because a small particle size implies high surface energy which is the driving force for sintering and porosity reduction.

Some features of the Ti-5.41Fe/Ni and Ti-7.57Fe/Ni powders are reported in Table 1 where it can be seen that their theoretical density is higher than that of pure Ti (i.e. $4.51 \mathrm{~g} / \mathrm{cm}^{3}$ ) because both Fe and $\mathrm{Ni}$, which are added as $\beta$ stabilisers, have higher density than Ti. From Table 1, both powders have a maximum particle size lower than $90 \mu \mathrm{m}$, oxygen content lower than $0.3 \mathrm{wt} . \%$ and nitrogen content lower than 0.03 wt.\%.

Table 1 Features of the Ti-5.41Fe/Ni and Ti-7.57Fe/Ni powders

\begin{tabular}{cccccc}
\hline \multirow{2}{*}{ Alloy } & Density $\left(\rho_{t h}\right)\left[\mathrm{g} / \mathrm{cm}^{3}\right]$ & Particle size $[\mu \mathrm{m}]$ & \multicolumn{3}{c}{ Interstitials [wt.\%] } \\
\cline { 2 - 6 } & & $D_{90}$ & $D_{M A X}$ & $O$ & $N$ \\
\hline Ti-5.41Fe/Ni & 4.70 & 88.52 & $<90$ & 0.29 & 0.026 \\
Ti-7.57Fe/Ni & 4.77 & 86.45 & $<90$ & 0.27 & 0.022 \\
\hline
\end{tabular}

After the uniaxial compacting at $700 \mathrm{MPa}$, the Ti-5.41Fe/Ni and Ti-7.57Fe/Ni powders had green densities of $77.1 \%$ and $77.5 \%$, respectively, where the compressibility of the Ti-7.57Fe/Ni powder was somewhat better. This is due to the effect of the fine $\mathrm{Fe} / \mathrm{Ni}(85-15)$ powder, which permitted a little bit better packing of the powder particles.

The variation of the density of the Ti-5.41Fe/Ni and Ti-7.57Fe/Ni alloys with the sintering temperature and the correlative trend of the total residual porosity left after the sintering step are shown in Fig. 2.

As typical in cold uniaxial pressed products, the density increases and the total residual porosity decreases along with the increment of the sintering temperature (Fig. 2). This is because the thermal energy induces the densification and the diffusion of the alloying elements. The system tries to reach a state of minimum energy by reducing the empty space constituted by the porosity left during the shaping of the powder and the interparticle and/or boundaries which are zones of high instability. In both cases (density and porosity) the greatest change is observed when the sintering temperature is increased from $900^{\circ} \mathrm{C}$ to $1200^{\circ} \mathrm{C}$ due to the combined fact that $900^{\circ} \mathrm{C}$ is a relatively low sintering temperature, most probably lower than the $\beta$ transus of the Ti-5.41Fe/Ni and Ti-7.57Fe/Ni alloys, and diffusion increases with the temperature.
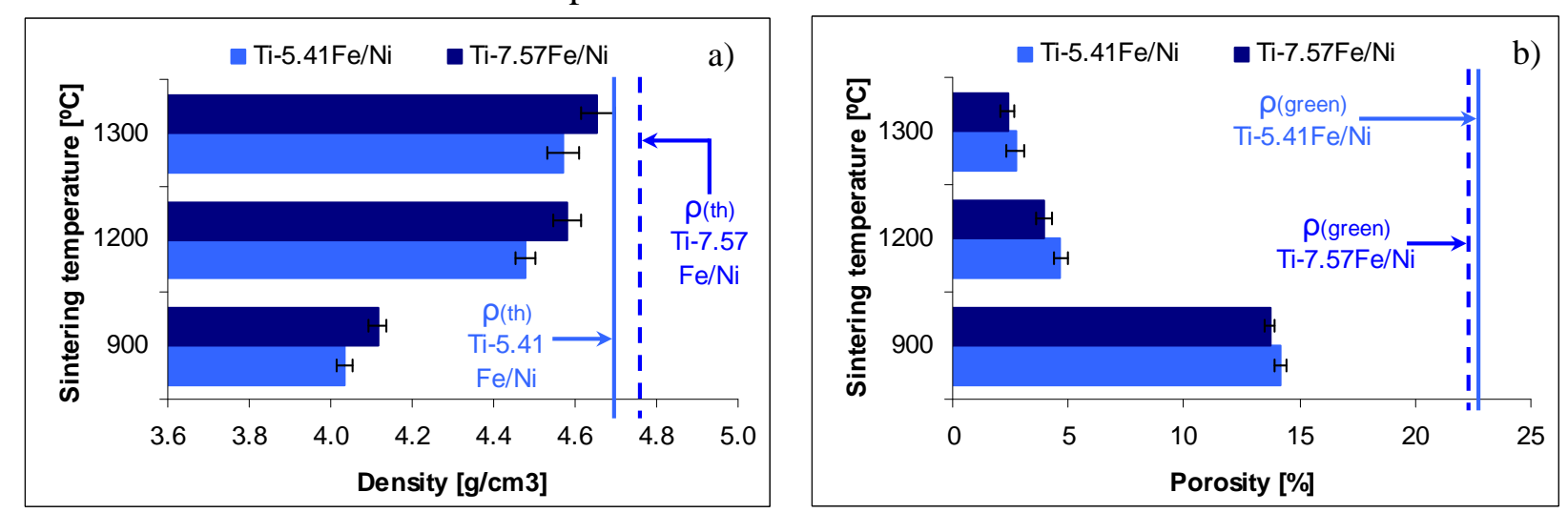

Figure 2 Variation of the density (a) and total residual porosity (b) of the Ti-5.41Fe/Ni and Ti7.57Fe/ $\mathrm{Ni}$ alloys versus sintering temperature

By comparing the behaviour of the two alloys, it can be noticed that the Ti-7.57Fe/Ni alloy reached higher density, which is due to the combined effect of the intrinsic higher green density of the alloy due to the greater amount of heavy alloying elements and the higher densification due to the higher amount of smaller particles as the porosity (i.e. relative density) data of Fig. 2 b) confirms. Similar behaviour with the sintering temperature and comparable level of porosity to those shown in Fig. 2 can be obtained in powder metallurgy $\alpha+\beta \mathrm{Ti}$ alloys by alloying elemental $\mathrm{Ti}$ with conventional master alloys $[10,11]$ or iron $[12,13]$ and stainless steel powder [7].

Fig. 3 reports the optical micrographs of the Ti-5.41Fe/Ni and Ti-7.57Fe/Ni alloys sintered at 
$1200^{\circ} \mathrm{C}$ and $1300^{\circ} \mathrm{C}$. It is worth mentioning that the microstructures of the materials sintered at $900^{\circ} \mathrm{C}$ are not fully homogeneous and small undissolved $\mathrm{Fe} / \mathrm{Ni}(85-15)$ powder particles of 3-5 $\mu \mathrm{m}$ were still present as microstructural feature.
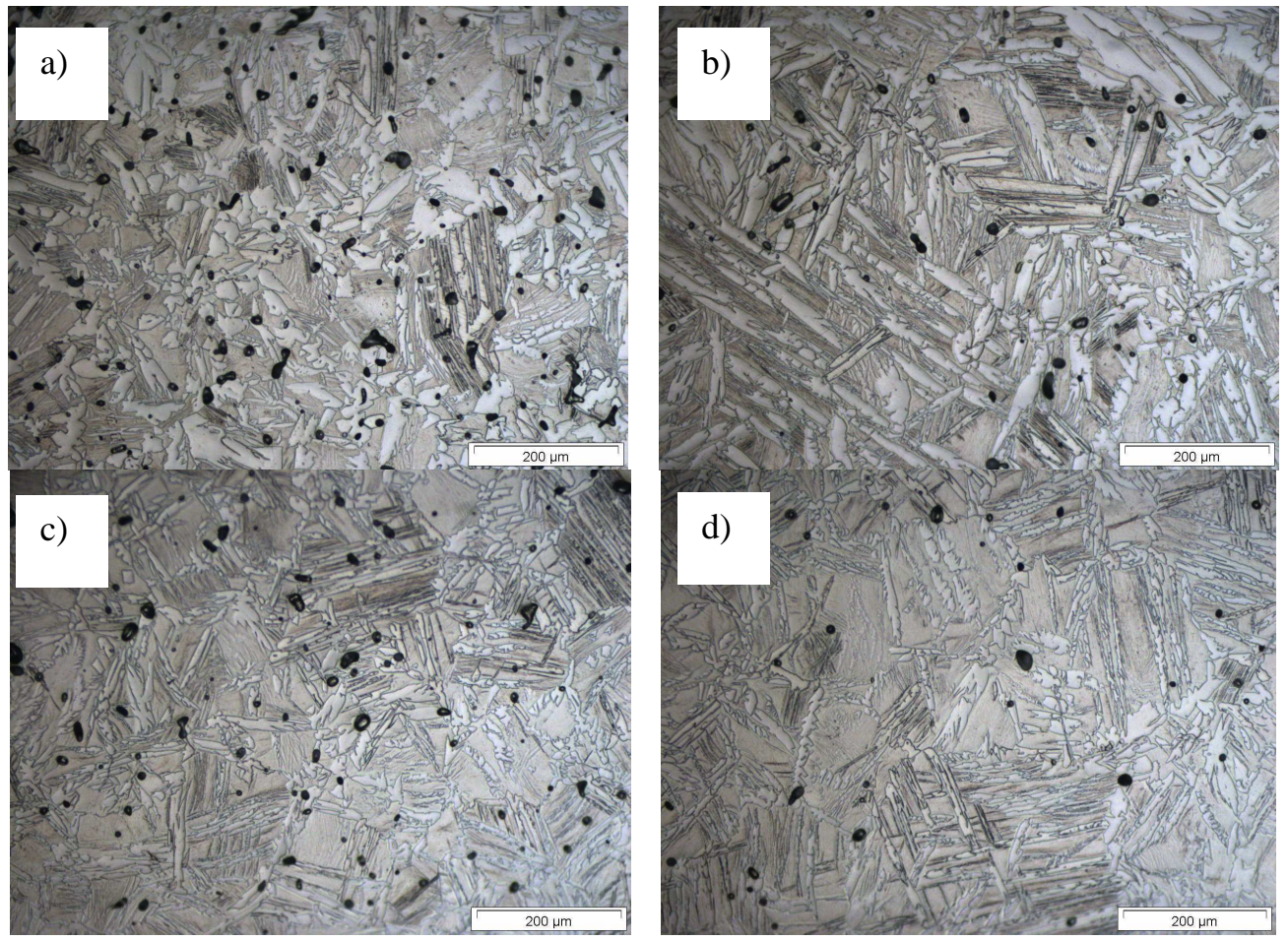

Figure 3 Optical micrographs of the Ti-5.41Fe/Ni (left) and Ti-7.57Fe/Ni (right) alloys, respectively, sintered at: a) and b) $1200^{\circ} \mathrm{C}$ and c) and d) $1300^{\circ} \mathrm{C}$

From the micrographs shown in Fig. 3 it can be seen that the microstructure of the sintered Ti$5.41 \mathrm{Fe} / \mathrm{Ni}$ and $\mathrm{Ti}-7.57 \mathrm{Fe} / \mathrm{Ni}$ alloys is compositionally homogeneous and it is composed of alpha grains and $\alpha+\beta$ lamellae, due to the stabilising effect of the alloying elements (i.e. Fe and $\mathrm{Ni}$ ). This is the typical microstructure of $\alpha+\beta$ Ti alloys slow cooled from a processing temperature above the $\beta$ transus of the alloy where Ti atoms, which occupy the position corresponding to a body-centred cubic structure $(\beta)$, starts to pack into the hexagonal crystals $(\alpha)$ rejecting the alloying elements whose local concentration increase and stabilises the $\beta$ Ti phase. From the micrographs of Fig. 3 it can also be noticed that the residual porosity left from the sintering process is mainly located at the grain boundaries and the pores are spherical in shape. No significant differences can be highlighted between the microstructural features of the two materials, if not that the relative amount of $\beta$ phase present in the Ti-7.57Fe/Ni alloy is greater than that of the Ti-5.41Fe/Ni alloy.

The variation of the Vickers hardness of the Ti-5.41Fe/Ni and Ti-7.57Fe/Ni alloys with the sintering temperature is given in Fig. 4.

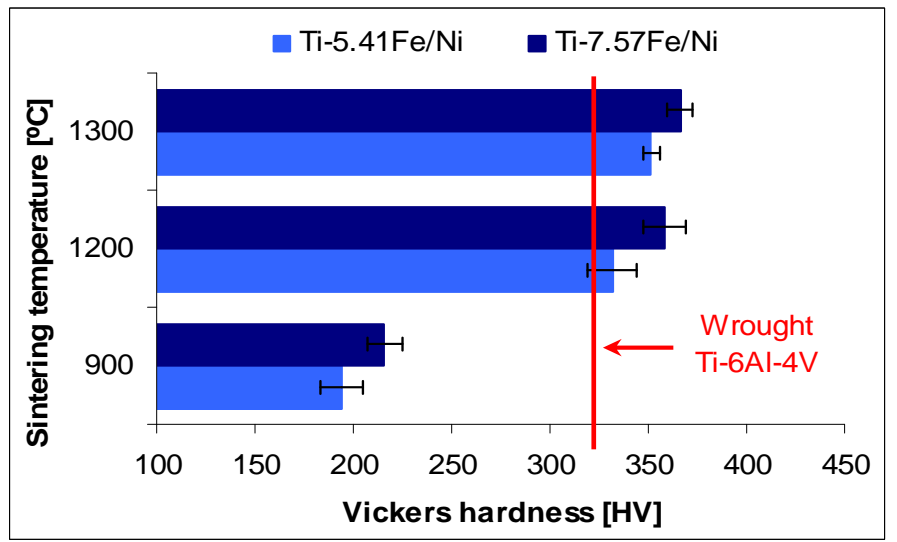

Figure 4 Variation of the hardness of the Ti-5.41Fe/Ni and Ti-7.57Fe/Ni alloys versus sintering temperature 
As in the case of the density, the hardness of the Ti-5.41Fe/Ni and Ti-7.57Fe/Ni alloys increases with the increment of the sintering temperature as a direct consequence of the reduction of the total residual porosity and homogenisation of the alloying elements. Both these two aspects, along with the higher solution hardening effect, also justify the slightly higher hardness of the Ti-7.57Fe/Ni alloy with respect to the Ti-5.41Fe/Ni alloy. The hardness of the Ti-5.41Fe/Ni and Ti-7.57Fe/Ni alloys is similar to that of conventional [14] and other low-cost Ti alloys $[7,13,15,16]$ obtained via PM methods. From Fig. 4 it is also clear that, despite the presence of some residual porosity as microstructural feature, the Fe/Ni alloyed materials have higher hardness than the wrought Ti-6Al$4 \mathrm{~V}$ alloy processed by conventional metallurgy. This is due to the hardening effect of oxygen in $\mathrm{Ti}$ where the Ti-5.41Fe/Ni and Ti-7.57Fe/Ni alloys have higher content, $0.3 \mathrm{wt} . \%$ in the starting powders, in comparison to the standard value of $0.2 \mathrm{wt} \%$ of the wrought Ti-6Al-4V alloy.

\section{Conclusions}

By means of studying the simultaneous employment of the cheapest powder metallurgy route of cold uniaxial pressing and sintering with the alloying of elemental $\mathrm{Ti}$ with fine $\mathrm{Fe} / \mathrm{Ni}$ (85-15) powder, it can be concluded that low-cost $\alpha+\beta$ PM Ti alloys can be obtained through this approach. In particular, the low-cost $\alpha+\beta$ PM Ti alloys fabricated with the addition of Fe/ $\mathrm{Ni}$ are characterised by similar sintering behaviour both in terms of density/total residual porosity and microstructural evolution to $\alpha+\beta$ Ti alloy with standard composition such as the Ti-6Al-4V alloy.

It is worth mentioning that the employment of fine powder particle for the alloying elements is advantageous because it does not significantly affect the compressibility and favours the densification of the material and the homogenisation of the same alloying elements.

\section{Acknowledgments}

The authors are thankful for the financial support from New Zealand Ministry of Business, Innovation and Employment (MBIE) through the UOWX1402 research contract.

\section{References}

[1] F.C. Campbell, Manufacturing Technology for Aerospace Structural Materials, 1st ed., Elsevier, 2006.

[2] I.J. Polmear, Light Alloys. Metallurgy of the Light Metals, 2nd ed., Edward Arnold, 1989.

[3] R.I. Jaffee, Physical metallurgy of titanium alloys, Progress in Metal Physics, 7 (1958) 65-163.

[4] F.H. Froes, M.N. Gungor, M.A. Imam, Cost-affordable titanium: the component fabrication perspective, JOM, 59 (2007) 28-31.

[5] R.M. German, Powder Metallurgy Science, 2nd ed., MPIF, Princeton, USA, 1994.

[6] F.C. Holden, H.R. Ogden, R.I. Jaffee, Heat treatment and mechanical properties of Ti-Fe alloys, Trans. Am. Inst. Min. Metall Eng. 206 (1956) 521-528.

[7] L. Bolzoni, E. Herraiz, E.M. Ruiz-Navas, E. Gordo, Study of the properties of low-cost powder metallurgy titanium alloys by 430 stainless steel addition, Mater. Des. 60 (2014) 628-636.

[8] D.B. Lee, K.B. Park, H.W. Jeong, S.E. Kim, Mechanical and oxidation properties of Ti-xFe-ySi alloys, Mater. Sci. Eng. A. 328 (2002) 161-168.

[9] K. Molchanova, Phase diagrams of titanium alloys, translation of atlas diagram (sostoyaniya Titanovyk Splavov), Israel Program for Scientific Translations, Jerusalem, (1965) 154.

[10] Y.F. Yang, S.D. Luo, G.B. Schaffer, M. Qian, Sintering of Ti-10V-2Fe-3Al and mechanical properties, Mater. Sci. Eng. A. 528 (2011) 6719-6726. 
[11] L. Bolzoni, P.G. Esteban, E.M. Ruiz-Navas, E. Gordo, Mechanical behaviour of pressed and sintered titanium alloys obtained from master alloy addition powders, J. Mech. Behav. Biomed. Mater 15 (2012) 33-45.

[12] C.S. Wang, K.S. Zhang, H.J. Pang, Y.Z. Chen, C. Dong, Laser-induced self-propagating reaction synthesis of Ti-Fe alloys, J Mater Sci. 43 (2008) 218-221.

[13] H. Hotta, M. Abe, T. Kuji, H. Uchida, Synthesis of Ti-Fe alloys by mechanical alloying, J. Alloys Compd. 439 (2007) 221-226.

[14] L. Bolzoni, E.M. Ruiz-Navas, E. Gordo, Flexural Properties, Thermal conductivity and electrical resistivity of prealloyed and master alloy addition powder metallurgy Ti-6Al-4V, Mater. Des. 52 (2013) 888-895.

[15] D. Kuroda, H. Kawasaki, A. Yamamoto, S. Hiromoto, T. Hanawa, Mechanical properties and microstructures of new Ti-Fe-Ta and Ti-Fe-Ta-Zr system alloys, Mater. Sci. Eng. C. 25 (2005) 312320.

[16] D.J. Lin, C.P. Ju, J.H.C. Lin, structure and properties of cast Ti-Fe alloys, transactions of the american foundrymen's society, 107 (1999) 859-864. 